\title{
370.
}

\section{ON THE SIGNIFICATION OF AN ELEMENTARY FORMULA OF SOLID GEOMETRY.}

[From the Philosophical Magazine, vol. xxx. (1865), pp. 413, 414.]

THE expression for the perpendicular distance of a point $(x, y, z)$ from a line through the origin inclined at the angles $(\alpha, \beta, \gamma)$ to the three axes respectively, is

$$
\begin{aligned}
p^{2}= & x^{2}+y^{2}+z^{n}-(x \cos \alpha+y \cos \beta+z \cos \gamma)^{2} \\
= & (y \cos \gamma-z \cos \beta)^{2} \\
& +(z \cos \alpha-x \cos \gamma)^{2} \\
& +(x \cos \beta-y \cos \alpha)^{2} ;
\end{aligned}
$$

and the remark in reference to it is that, if at the given point $P$ we draw, perpendicular to the plane through $P$ and the given line, a distance $P K$ equal to the distance of $P$ from the given line, then the expressions

$$
y \cos \gamma-z \cos \beta, \quad z \cos \alpha-x \cos \gamma, \quad x \cos \beta-y \cos \alpha,
$$

which enter into the preceding formula, denote respectively the coordinates of the point $K$ referred to $P$ as origin.

If the given line instead of passing through the origin pass through the point $x_{0}, y_{0}, z_{0}$, then the corresponding expressions are of course

$$
\left(y-y_{0}\right) \cos \gamma-\left(z-z_{0}\right) \cos \beta, \quad\left(z-z_{0}\right) \cos \alpha-\left(x-x_{0}\right) \cos \gamma, \quad\left(x-x_{0}\right) \cos \beta-\left(y-y_{0}\right) \cos \gamma,
$$

and if we denote the "six coordinates" of the given line, viz. by

$\cos \alpha, \quad \cos \beta, \quad \cos \gamma, \quad y_{0} \cos \gamma-z_{0} \cos \beta, \quad z_{0} \cos \alpha-x_{0} \cos \gamma, \quad x_{0} \cos \beta-y_{0} \cos \gamma$,

$$
a, b, c, \quad f \quad, \quad, \quad h
$$


respectively (so that $a f+b g+c h=0$ ), then the three expressions become

$$
c y-b z-f, \quad a z-c x-g, \quad b x-a y-h
$$

respectively.

It is moreover clear that if the point $P$ be moved to $P^{\prime}$ by an infinitesimal rotation $\omega$ about the given line, then $P^{\prime}$ lies on the line $P K$ at a distance $P P^{\prime},=\omega P K$, from the point $P$, and the displacements of $P$ in the directions of the axes are consequently equal to

$$
\omega(c y-b z-f), \quad \omega(a z-c x-g), \quad \omega(b x-a y-h)
$$

respectively, which is a fundamental formula in the theory of the infinitesimal rotations of a solid body.

Cambridge, October 26, 1865. 